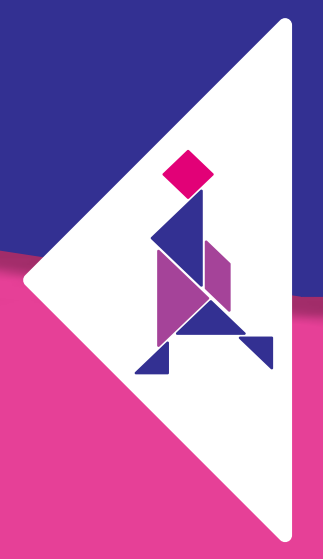

\title{
Exercício físico e capacidade cognitiva em idosos
}

\author{
Physical exercise and cognitive \\ capacity of older adults
}

FisiSenectus. Unochapecó Ano 6, n. 2- Jul/Dez. 2018 p. $45-51$

Marzo Edir Da Silva Grigoletto. dasilvame@gmail.com

Departamento de Educação Física, Centro de Ciências Biológicas e da Saúde, Universidade Federal de Sergipe (UFS), Brasil.

Antônio Gomes de Resende-Neto.neto.resende-edf@hotmail.com

Departamento de Educação Física, Centro de Ciências Biológicas e da Saúde, Universidade Federal de Sergipe (UFS), Brasil.

Juan Manuel Garcia-Manso. jgarciamanso@gmail.com

Departamento de Educación Física, Universidad de Las Palmas de Gran Canaria (ULPGC), España

\section{Resumo}

Introdução: Atualmente, uma das terapias mais eficazes e de baixo custo para reduzir os efeitos incapacitantes das doenças neurodegenerativas em idosos é o exercício físico. Entretanto, pouco se sabe sobre seus mecanismos de ação e benefícios sobre a Reserva Cognitiva (RC). Objetivo: Apresentar e discutir estudos sobre os efeitos do exercício físico sobre a morfologia e funcionalidade do cérebro humano e sugerir uma estruturação de programas para melhora da RC em idosos. Método: Trata-se de um artigo especial de opinião fundamentado em pesquisas nas bases de dados MEDLINE e Scholar Google, a fim de abordar os seguintes aspectos: (a) benefícios do exercício físico na RC e (b) como estruturar sessões respeitando os critérios de eficácia e funcionalidade. Resultados: Os principais benefícios do exercício estão relacionados ao aumento do fluxo sanguíneo cerebral, redução dos fatores risco cardiovascular, melhora da plasticidade sináptica e aumento no número de fatores de crescimento do cérebro. Dentre os aspectos a serem considerados na elaboração de tarefas específicas para estimulação cognitiva, temos: a análise do perfil de comprometimento neuropsicológico do indivíduo; a complexidade dos padrões de movimento; o estímulo a diferentes componentes da aptidão física e a análise subjetiva em termos de bem-estar psicológico e autoestima. Conclusão: Embora este artigo aponte para os efeitos positivos do exercício físico como uma estratégia eficaz no tratamento e na prevenção de doenças neurodegenerativas, pode-se afirmar que existe uma lacuna no campo do conhecimento acerca dos efeitos da atividade e/ou exercício físico a sobre a RC.

\section{Palavras-chave}

Envelhecimento; Cognição; Atividades diárias; Qualidade de vida.

\section{Fisiß̌enectus}




\begin{abstract}
Introduction: Currently, one of the most cost-effective and effective therapies to reduce the disabling effects of neurodegenerative diseases in the elderly is exercise. However, little is known about its mechanisms of action and benefits over Cognitive Reserve (CR). Objective: To present and discuss studies on the effects of physical exercise on the morphology and functionality of the human brain and to suggest a structuring of programs to improve cognitive reserve in the elderly. Method: This is a special opinion article based on research in the MEDLINE and Google Scholar databases, in order to address the following aspects: (a) benefits of physical exercise in CR and (b) how to structure sessions in accordance with the criteria of effectiveness and functionality. Results: The main benefits of exercise are related to increased cerebral blood flow, reduction of cardiovascular risk factors, improvement of synaptic plasticity and increase in the number of brain growth factors. Among the aspects to be considered in the elaboration of specific tasks for cognitive stimulation we have: the analysis of the profile of neuropsychological impairment of the individual; the complexity of movement patterns; the stimulus to different components of physical fitness and subjective analysis in terms of psychological well-being and self-esteem. Conclusion: Although this article points to the positive effects of physical exercise as an effective strategy in the treatment and prevention of neurodegenerative diseases, it can be affirmed that there is a gap in the field of knowledge about the effects of physical activity and/or exercise on the RC.
\end{abstract}

Keywords

Aging; Cognition; Activities of daily living.

\section{Introdução}

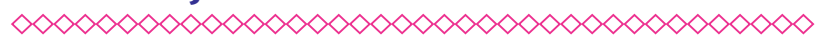

0 aumento da expectativa de vida representa um progressivo envelhecimento populacional (aproximadamente 900 milhões de pessoas têm mais de 60 anos) alterando a pirâmide etária de muitas sociedades. Enquanto no ano de 1900 havia entre 10 e 17 milhões de pessoas com mais de 65 anos ( $\approx 1 \%$ da população mundial), no ano de 2050 esse número pode crescer até 25 milhões $(\approx 20 \%)^{1}$.

Ainda que, nos últimos anos, se tenha registrado importantes avanços no esclarecimento sobre processos moleculares que afetam nossa mente a partir da senescência, o caminho a percorrer ainda é grande. As alterações morfológicas (neurotransmissores, neurônios, células gliais, sinapses e vasos sanguíneos) e funcionais podem desencadear a deterioração da função cognitiva em um processo explicado, em parte, pela atrofia dos tecidos neurais do hipocampo e pelo aumento dos fatores de risco cardiovascular, que são responsáveis pelo decréscimo progressivo do metabolismo e do fluxo sanguíneo cerebral, relacionando-se com doenças neurodegenerativas, como Alzheimer, Parkinson e outros tipos de demência que também podem causar alterações de humor e de personalidade².

Além do mais, a dificuldade de armazenar e resgatar informações, gerada pelo déficit cognitivo, pode causar prejuízos sociais e ocupacionais ao idoso, como perda da autoestima, o autoabandono e o isolamento social ${ }^{3}$. Também se acredita que a RC caracterizada pelas capacidades intelectuais que uma pessoa acumula ao longo da vida por meio de experiências pessoais, culturais, acadêmicas e recreativas é reduzida ao longo dos anos mesmo em indivíduos saudáveis ${ }^{4}$.

Nessa perspectiva, para encontrar uma solução eficiente e definitiva, os profissionais da saúde estão desenvolvendo estratégias que visam a reduzir e/ou retardar os incapacitantes efeitos destas doenças e aumentar a capacidade cognitiva em pessoas idosas. Na ausência de terapias farmacológicas ou cirúrgicas, nos últimos anos, foram encontradas evidências científicas de que as intervenções psicossociais como atividades coletivas diversas estão diretamente ligadas à melhoria da qualidade de vida destes doentes e indiretamente na de seus cuidadores. Uma das terapias mais aceitas é o exercício físico, em especial, aquele 
ligado à estimulação cognitiva e/ou ativação de padrões motores com alguma complexidade coordenativa ${ }^{5}$.

Nesse contexto, as informações apresentadas nesse manuscrito podem proporcionar subsídios para orientação de exercícios físicos que, com base nas respostas funcionais neurais conhecidas, sejam mais úteis e assertivas para a melhora da funcionalidade e da qualidade de vida em idosos, a partir da compreensão dos efeitos do envelhecimento na cognição, dos possíveis benefícios do exercício e as principais características dos protocolos de intervenção direcionados exclusivamente para melhorar a função cognitiva.

\section{Efeito do exercício físico sobre a morfologia e funcionalidade do cérebro humano}

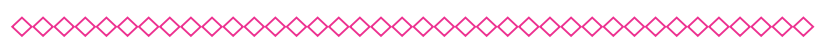

Qualquer movimento que envolva a ativação de diferentes estruturas cerebrais (centrais e periféricas) em função da complexidade motora do gesto e do seu objetivo, por mais básico que seja, de forma mais ou menos intensa, gera redes completas de informação que garantem a efetividade do movimento. Porém, a qualidade dos mesmos se vê alterada com o envelhecimento, tornando-os doloridos, lentos, ásperos e possivelmente com erros de planejamento e execução ${ }^{6}$. E este é o ponto onde entra a atividade física adaptada ao idoso com evidentes alterações cognitivas.

Portanto, de forma geral, é possível indicar que cada fase de um movimento implica diferentes zonas cerebrais especializadas, resumidas no Quadro $\mathbf{1}^{7}$.

É fato que o exercício físico aumenta a vascularização do cérebro, favorecendo a chegada de nutrientes ${ }^{3}$. Constantemente os vasos sanguíneos cerebrais alteram o fluxo de sangue em resposta às necessidades de mudanças momentâneas de cada zona. Isso é especialmente observado quando a tarefa tem certo grau de complexidade técnica e requer a participação de processos conscientes e mecanismos de controle motor para a execução ${ }^{5}$.
Em qualquer caso, não podemos esquecer que o cérebro de uma pessoa saudável se comporta de maneira diferente de um cérebro que apresenta alguma psicopatologia (esquizofrenia, autismo e depressão) ou alterações cognitivas evidentes (Alzheimer e demência senil). A resposta hemodinâmica cortical a diferentes estímulos (físicos, perceptivos ou cognitivos) proporcionam um sinal detectável que pode informar a presença e localização da ativação neural e das redes que são geradas ${ }^{8}$.

Medindo o fluxo sanguíneo cerebral (FSC), pode-se determinar indiretamente a atividade do cérebro, pois é regulado por diferentes vias após a liberação de neurotransmissores nas sinapses, causando ativação de receptores nos neurônios e astrócitos. Os neurônios controlam o FSC por meio da dilatação dos vasos sanguíneos por ação do processo: Receptores de N-metil-D-aspartato $\rightarrow$ Prostaglandinas $\rightarrow$ Sintase neuronal de óxido nítrico (nNOS). 0 astrócitos controlam a FSC por meio da dilatação dos vasos sanguíneos para a produção e liberação de metabólitos do ácido araquidônico: prostaglandinas $\rightarrow$ ácido epoxieicosatrienoico ${ }^{9}$.

Paralelamente a estes mecanismos, o exercício físico aumenta o número de fatores de crescimento do cérebro, especialmente: o fator neurotrófico derivado do cérebro, o fator de crescimento endotelial vascular e IGF-I. Todos esses processos estimulam a neurogênese e a melhora da plasticidade sináptica, favorecendo processos neuroprotetores de regeneração do sistema nervoso. E são aumentados no córtex motor quando as pessoas adquirem habilidades motoras advindas do exercício ${ }^{10}$.

\section{Efeito do exercício físico na Reserva Cognitiva}

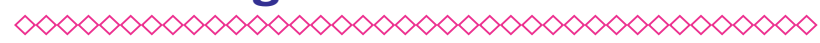

A RC também se descreve como a capacidade cerebral que nos permite tolerar melhor os efeitos associados a neuropatologias antes de chegar a um limiar de deterioração em que os sintomas clínicos começam a se manifestar ${ }^{4}$.

A participação em programas de exercícios físicos pode contribuir para melhora cognitiva do ser humano (Figura 1). Por essa razão, recentes 
pesquisas têm se centrado em estudar essa relação, concluindo que o exercício pode reduzir o risco de comprometimento cognitivo por meio da melhoria da $\mathrm{RC}^{11-16}$

Em estudo de revisão realizado por Rolland et al. ${ }^{11}$, os autores apontaram que o exercício físico pode melhorar a saúde cerebral, reduzir o declínio cognitivo e os efeitos adversos associados por meio de mecanismos como a redução dos fatores de risco cardiovascular (hipertensão, diabetes, hipercolesterolemia e obesidade), além de aumentar a synaptogenesis, neurogênese, mielenização, citoarquitetura cerebral e as propriedades eletrofisiológicas do sistema nervoso central. Também parece causar efeitos positivos sobre a memória e outros fatores cognitivos colaterais (noção espaço-temporal, visual, orientação racional, estado emocional, pessoal e social etc.) vinculados à capacidade cognitiva do sujeito ${ }^{13}$.

Yaffe et al. ${ }^{14}$ apontaram que indivíduos que se exercitam pelo menos três vezes por semana possuem um risco mais baixo de desenvolver demência quando comparado com menos ativos. Schuit et al. ${ }^{15}$, identificaram que idosos ativos ( $>1$ hora/ dia de exercício) possuíam um risco de comprometimento cognitivo duas vezes menor que os menos ativos (< 1 hora/dia). Weuve et al. ${ }^{16}$ asseguram que fazer pelo menos 15 minutos de exercícios físicos (alternando passeios, caminhadas, ciclismo, ginástica, natação, hidroginástica, musculação ou outras formas de exercício), três vezes por semana, reduz o risco de desenvolver demência. Estes resultados sugerem que o limiar de intensidade para reduzir o risco de deterioração cognitiva e demência pode ser baixo. No entanto, a intensidade ainda não está clara.

\section{Estruturação de programas com exercícios físicos para melhora da Reserva Cognitiva em idosos}

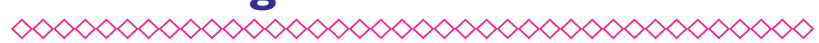

Dentre os aspectos a serem considerados na elaboração de tarefas específicas com exercício, o perfil de comprometimento neuropsicológico do indivíduo é um dos mais importantes. Portanto, é importante levar em consideração a perda de memória (a curto e longo prazo), disfunção em habilidades instrumentais (ex.: idioma e práxis) e deterioração de funções executivas importantes (ex.: planejamento de ações, organização de tarefas, complexidade espaço-temporais). A partir desse aspecto, criam-se condições para seleção de exercícios e atividades com níveis de complexidade motora e cognitiva executável, favorecendo a segurança e eficiência da intervenção.

0 American College of Sports Medicine ${ }^{17}$ sugere que o benefício da atividade física está relacionado com à quantidade de atividade por dia (gasto energético), e menos ao modo como é praticada. No entanto, na opinião dos autores deste manuscrito, o exercício físico deve possuir características especiais que garantem um efeito muito específico sobre as estruturas cognitivas e morfofuncionais do indivíduo.

Assim, qualquer programa que se fundamente em exercícios físicos para idosos com déficit cognitivo deve incluir atividades de: flexibilidade, equilíbrio, potência, força muscular e resistência. Aplicadas em exercícios com dupla-tarefa, variados, dinâmicos e de alta complexidade motora em ações multiarticulares, objetivando dificultar a lembrança e a reprodução dos movimentos, e assim, representar um constante desafio cognitivo e, consequentemente, um importante estímulo para melhoria da saúde mental ${ }^{18}$.

Além disso, no planejamento e na avaliação de programas de exercícios físicos deve levar em conta não só a relevância de um determinado tipo de exercício (aeróbico vs. anaeróbico), ou seu modelo de aplicação (intensidade, volume, densidade, frequência e duração), mas também a análise subjetiva em termos de bem-estar psicológico e autoestima. Por exemplo, uma sensação gratificante e imediata pode ajudar a melhorar a autoeficácia percebida, a sensação de controle e a criação de hábito para prática de exercício.

Por tanto, propomos de acordo com as características dos idosos com funções cognitivas prejudicadas, uma intervenção especializada onde a estimulação cognitiva em torno do afetivo e da variabilidade motora são tão importantes quanto a ação mecânica exercício, e está acompanhada de estímulos que forçam os indivíduos a ativar sua capacidade cognitiva mediante elementos como: 
- A evocação de memórias (ex.: lugar de nascimento e número de irmãos) ou imagens (ex.: sua casa, sua família e lugares frequentados) que são familiares e positiva. Neste ponto, é necessário conhecer profundamente o sujeito para evitar recordar episódios negativos para o seu estado emocional.

- A resolução de tarefas motoras e/ou cognitivas que lhes obriguem a manter-se fisicamente e mentalmente ativos enquanto estão realizando a atividade. Jogos coletivos são uma alternativa especialmente interessante.

- A incorporação de pessoas ao redor das atividades (especialmente parentes). Neste ponto, a participação em atividades paralelas (ex.: concertos, visitas culturais e competições esportivas), julgamos altamente integrativas e estimulantes.

- A familiarização com o ambiente de residência, com especial relevância para ambientes na natureza. Bem aos que estão próximos de sua residência ou os que requerem um pequeno deslocamento para acessá-los.

Desse modo, acredita-se que orientações de treinamento seriam destinadas ao desenvolvimento da coordenação (controle motor + integração sensorial), do equilíbrio, da mobilidade, da força muscular, da eficiência metabólica, da motricidade-cognitiva-evocativa, da motricidade-afetiva-socializadora, da atividade-familiar-aglutinadora da motricidade alternativa (ex.: danças, canções e jogos lúdicos).

\section{Considerações finais}

$\infty \times \infty \times \infty \times \infty \times \infty \times \infty \times \infty \times \infty \times \infty \times \infty \times \infty \times \infty \infty \infty$

Tudo parece indicar que o exercício físico pode ser considerado uma ferramenta eficaz e de baixo custo para retardar a perda natural da capacidade cognitiva em senis. Acredita-se, também, que programas com exercícios não devem ser utilizados somente como um mero instrumento lúdico, mas sim, ser composto por atividades com dupla tarefa, de alta variabilidade e complexidade motora para promover adaptações multisistêmicas e gerar um forte estímulo cognitivo. Além disso, afirmamos que a estimulação por evocação ou resolução de tarefas e o ambiente socioemocional em que ocorrem, são tão importantes quanto as características mecânicas e bioenergéticas das atividades.

\section{Referências}

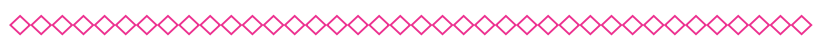

1. Olshansky SJ, Carnes BA, Cassel C.In search of Methuselah: estimating the upper limits to human longevity. Science, 1990;250(4981): 634-40.

2. Prince $M$, et al. World Alzheimer Report. The global impact of dementia. An analysis of prevalence, incidence, cost and trends. Alzheimer's Disease International, London, 2015.

3. Kirk-Sanchez NJ, Mcgough, EL. Physical exercise and cognitive performance in the elderly: current perspectives. Clin Interv Aging. 2014;18(9):51-62.

4. Scarmeas N, Stern Y. Cognitive reserve and lifestyle. J Clin Exp Neuropsychol. 2003;25(5):\} 625--33.

5. Colangeli S, Boccia M, Verde P, Guariglia P, Bianchini F, Piccardi L. Cognitive Reserve in Healthy Aging and Alzheimer's Disease: A MetaAnalysis of fMRI Studies. Am J Alzheimers Dis Other Demen. 2016; 31(5):443-9.

6. Hunter SK, Pereira HM, Keenan KGThe aging neuromuscular system and motor performance. J Appl Physiol. 2016;121(4):982-95.

7. Hillman EM, Devor A, Bouchard MB, Dunn AK, Krauss GW, Skoch J, et al. Depth-resolved optical imaging and microscopy of vascular compartment dynamics during somatosensory stimulation. Neuroimage. 2007;(1):89-104.

8. Woolsey TA, Rovainen CM, Cox SB, Henegar $\mathrm{MH}$, Liang GE, Liu D, et al. Neuronal units linked to microvascular modules in cerebral cortex: response elements for imaging the brain. Cereb Cortex. 1996; 6(5):647-60.

9. Bélanger M, Magistretti PJ. The role of astroglia in neuroprotection. Dialogues in clinical neuroscience. 2009;11(3): 281-95. 
10. Maass A, Düzel S, Brigadski T, Goerke $M$, Becke A, Sobieray U, et al. Relationships of peripheral IGF-1, VEGF and BDNF levels to exercise-related changes in memory, hippocampal perfusion and volumes in older adults. NeuroImage. 2016;1(131):142-54.

11. Rolland Y, Abellan Van Kan G, Vellas B. Physical Activity and Alzheimer's Disease: From Prevention to Therapeutic Perspectives. J Am Med Dir Assoc. 2008;9(6):390-405.

12. Dik M, Deeg DJ, Visser M, Jonker C.Early life physical activity and cognition at old age. J Clin Exp Neuropsychol. 2003;25(5):643-53.

13. Ide K, Secher, NH. Cerebral blood flow and metabolism during exercise. Progress in neurobiology. 2000;61(4):397-414.

14. Yaffe K, Barnes D, Nevitt M, Lui LY, Covinsky K. A prospective study of physical activity and cognitive decline in elderly women: Women who walk. Arch Intern Med. 2001;161(14):1703-8.

15. Schuit AJ, Feskens EJ, Launer LJ, Kromhout D. Physical activity and cognitive decline, the role of the apolipoprotein e4 allele. Med Sci Sports Exerc. 2001;33(5):772-7.

16. Weuve J, Kang JH, Manson JE, Breteler MM, Ware JH, Grodstein F.Physical Activity, Including Walking, and Cognitive Function in Okder Women. Journal American Medical Association. 2004;292(12):1454-61.

17. AMERICAN COLLEGE OF SPORTS MEDICINE, ACSM's guidelines for exercise testing and prescription. Lippincott Williams \& Wilkins, 2013.

18. Resende-Neto AG, Silva-Grigoletto ME da, Santos MS, Cyrino ES. Treinamento funcional para idosos: uma breve revisão. R. Bras. Ci. e Mov. 2016;24(3):167-77. 


\section{Anexos}

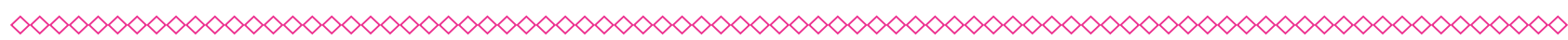

Quadro 1 - Fase de um movimento e suas implicações nas zonas cerebrais especializadas.

- Concentração: ativação do tálamo;

- Eliminação de elementos irrelevantes: ativação do lobo frontal;

- Interpretação do meio ambiente: ativação do lobo parietal;

- Ativação da memória: ativação do putâmen (gânglios basais);

- Desenvolvimento do plano de ação: ativação do córtex pré-motor;

- Percepção consciente do padrão de execução:

- Instruções de execução: Córtex pré-motor $\rightarrow$ Córtex motor $\rightarrow$ Medula espinal $\rightarrow$ Motoneurônios alfa;

- Ação consciente e correção de movimento: Cerebelo.

(clique para voltar ao texto)

Figura 1 - Aumento da Reserva Cognitiva (RC) e o declínio contínuo do desempenho cognitivo global com e sem o exercício físico na população idosa.

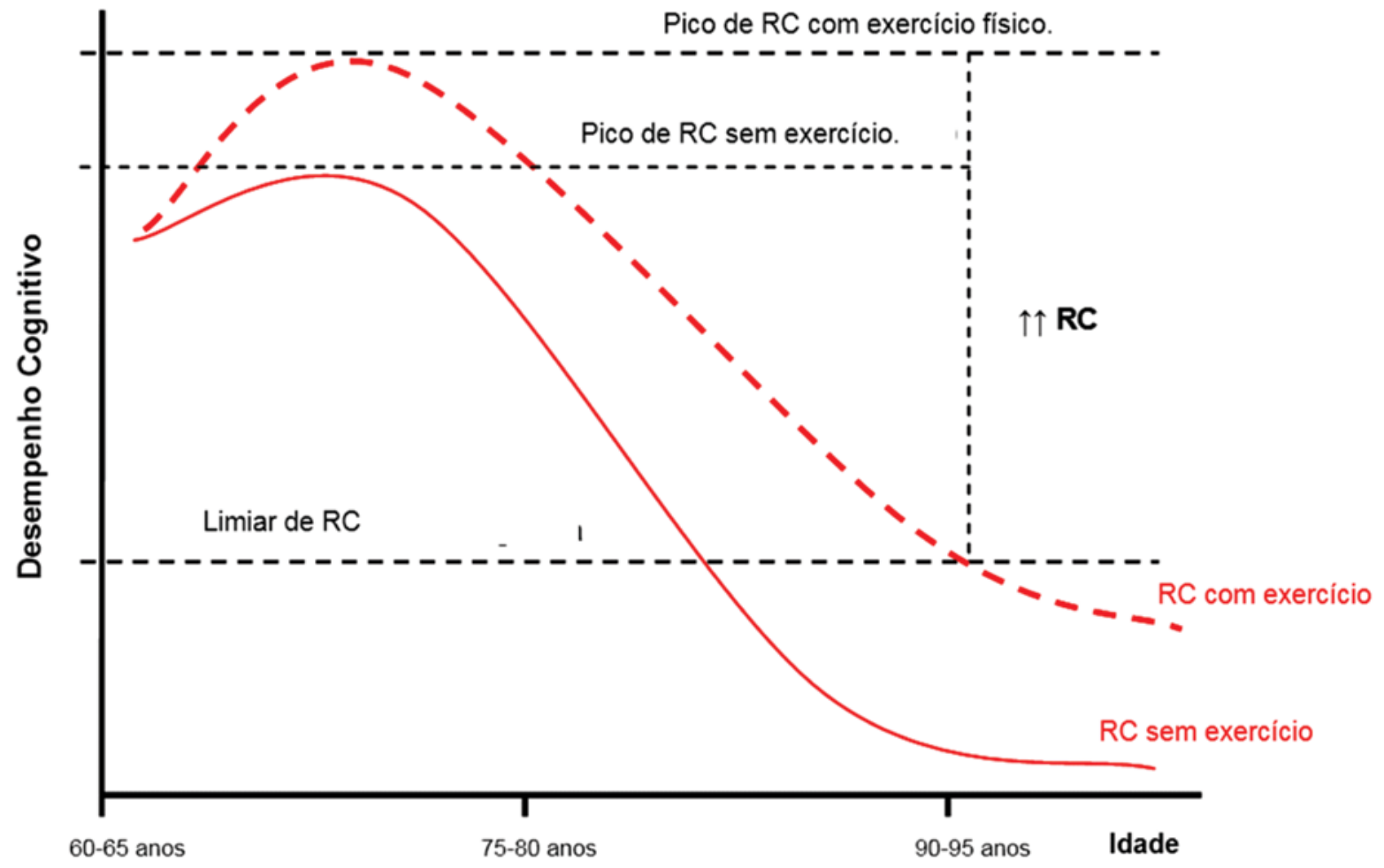

Fonte: Elaborada pelos autores.

(clique para voltar ao texto) 